\title{
Intravenous Fluid Management in the Pediatric Hospital Setting: Is Isotonic Fluid the Right Approach for all Patients?
}

Jonathan B. Edelson, $M D^{1,3, *}$

Evan W. Orenstein, $M D^{1}$

Lisa B. Zaoutis, $M D^{1}$

Lawrence Copelovitch, $M D^{1,2}$

\author{
Address \\ ${ }^{*}$ DDepartment of Pediatrics, Children's Hospital of Philadelphia, Philadelphia, PA, \\ USA \\ Email: edelsonj@email.chop.edu \\ ${ }^{2}$ Children's Hospital of Philadelphia, Division of Nephrology, Philadelphia, PA, \\ USA \\ ${ }^{3}$ Residency Education Office, 3401 Civic Center Blvd, Philadelphia, Pa 19104, USA
}

Published online: 7 January 2015

(C) Springer International Publishing AG 2015

This article is part of the Topical Collection on Hospital Medicine

Keywords Intravenous fluid · Hypotonic fluid - Isotonic fluid - Hyponatremia - Children · Fluid overload •

Antidiuretic hormone (ADH) · Syndrome of inappropriate antidiuresis (SIAD)

\section{Opinion statement}

The purpose of the study is to review the recent controversy in the optimal tonicity of maintenance intravenous fluids for pediatric patients and to suggest strategies for the selection of maintenance fluid rate and composition for the pediatric hospitalist. The goals of maintenance intravenous fluid management are to preserve adequate effective circulating volume, maintain normal electrolytes, and avoid fluid overload. Wide practice variation exists in the provision of maintenance fluids for pediatric patients admitted to the general ward. Two recent meta-analyses have favored isotonic over hypotonic maintenance fluids to avoid iatrogenic hyponatremia. However, the generalizability of these findings are limited as randomized studies have focused on postoperative patients and ICU settings, comparisons in terms of fluid composition have been widely variable, few studies have examined the rate of fluid administration, and there has been insufficient examination of potential adverse effects of increased solute load. In the midst of such controversy, the pediatric hospitalist must critically evaluate current evidence when choosing 
maintenance fluids. To mitigate iatrogenic hyponatremia while simultaneously minimizing the risks of possible excess solute, we propose using physiologic concepts including intravascular volume at the time of initiation of maintenance fluids, initial serum sodium, and risk for increased secretion of antidiuretic hormone (ADH) to guide maintenance fluid choice.

\section{Introduction}

Of the over two million children admitted to US hospitals every year, a great many require intravenous (IV) fluids during their hospitalization. The goals of maintenance IV fluid therapy are to maintain adequate effective circulating volume, maintain normal electrolytes, and avoid fluid overload. Given the impact on multiple physiologic variables, it is not surprising that wide practice variation exists $[1,2]$ with ongoing and active debate over the correct strategy for maintenance IV fluid administration in different disease states.

The basis for our modern concepts of maintenance fluid calculations originated in the work of Holliday and Segar in 1957 [3] who suggested that in otherwise healthy children, daily fluid requirements and losses correlate directly with caloric needs and therefore are indirectly related to the patient's weight and size. They reasoned that all fluid losses were derived from insensible losses, basal energy expenditure, and urine formation. Further, they estimated the electrolyte composition required to maintain homeostasis based on the content found in both human and cow's milk. The result of these water and electrolyte calculations led to the use of hypotonic fluids as the mainstay of maintenance IV fluids used in pediatric patients over the last 50 years. Even in a recent study, pediatric residents selected hypotonic fluids in $78 \%$ of simulated cases designed to suggest elevated antidiuretic hormone (ADH) levels [2].

Over the last decade, it is has become increasingly well recognized that the pathophysiologic changes in sick children perturb normal homeostatic mechanisms in ways that question the appropriateness of applying the classic Holliday and Segar approach. In particular, many children have both osmotic and non-osmotic stimuli for increased ADH production, leading to the concern for the development of hyponatremia which can be exacerbated by the use of hypotonic maintenance fluids. Two recent metaanalyses $[4 \bullet, 5 \bullet \bullet]$ of randomized trials comparing hypotonic and isotonic maintenance fluids in pediatric populations found that the use of hypotonic fluids was associated with $>2$-fold increased risk of developing serum sodium $(\mathrm{pNa})<135 \mathrm{mEq} / \mathrm{L}$ and $>5$-fold increased risk of $\mathrm{pNa}<130 \mathrm{mEq} / \mathrm{L}$. While these studies are salient, it is important to note that the data have several important limitations which preclude a blanket recommendation for the use of isotonic fluids in all settings at this time. At least $67 \%$ of all randomized subjects were postoperative surgical patients, and the majority of all studies were completed in ICU settings, limiting generalizability particularly to medical patients on inpatient pediatric wards. Furthermore, the types of hypotonic fluids used were widely variable across studies, thereby limiting comparisons. In addition, the studies were generally underpowered and not designed to detect disease-specific risks of developing hyponatremia. Very few studies have examined the impact of changes in volume administration [6-8]. While most studies checked for hypernatremia, few explicitly looked for other adverse events associated with an increased solute load, particularly fluid overload [9] or hyperchloremic metabolic acidosis [10-12].

In the midst of such controversy, how should pediatric hospitalists prescribe maintenance IV fluids? In lieu of a one-size-fits-all strategy, we propose using physiologic concepts to guide initial maintenance fluid choice. In this review, we discuss recent evidence with regard to tonicity and rate of maintenance fluids, propose an algorithm to direct maintenance fluid rate and composition, and suggest future directions for research. 


\section{The Controversy}

While the use of hypotonic fluids based on the work of Holliday and Segar remained doctrine through the latter half of the twentieth century, Moritz and Ayus [13] began to challenge conventional wisdom after compiling $>50$ case reports of neurological morbidity associated with hospital-acquired hyponatremia in the setting of hypotonic fluid use. Similar reviews [14] led to observational and eventually randomized studies comparing hypotonic to isotonic fluids in select populations, culminating most recently in two metaanalyses $[4 \bullet 5 \bullet \bullet]$ favoring the use of isotonic maintenance fluids.

These studies argue strongly against the use of hypotonic maintenance fluids across all pediatric populations, as emphasized by the safety warning issued by the UK's National Patient Safety Agency and supported by the Royal College of Paediatrics [15]. However, as detailed above, limitations in the evidence including the populations studied, study heterogeneity, and paucity of data on potential adverse events from an increased solute load as well as the effect of changes in administration rate preclude a simple transition to isotonic fluids for all-comers. The focus on tonicity of maintenance fluid without adequate study of the rate or volume of administration is concerning in the context of our current physiological understanding of the causes of hospital-acquired hyponatremia.

At least three randomized studies have examined the impact of the rate of maintenance fluid administration on hyponatremia and other outcomes. The relatively small sample sizes, varied comparisons, and limited populations make this area highly understudied, particularly with regard to patients admitted to the general pediatrics ward. In Kannan et al. [6], there was no statistically significant difference in the rate of hyponatremia comparing 58 patients receiving $5 \%$ dextrose and $0.9 \%$ saline at full maintenance rate to 53 patients receiving $5 \%$ dextrose and $0.18 \%$ saline at $2 / 3$ of the maintenance rate (RR 2.2, $95 \%$ CI $0.2-23.5$ for $\mathrm{pNa}<130 \mathrm{mEq} / \mathrm{L}$; RR 1.1, 95\%CI 0.3-3.6 for $\mathrm{pNa}<$ $135 \mathrm{mEq} / \mathrm{L})$. In Yung and Keeley [8], 50 critical care patients were randomized into four treatment groups ( $0.9 \%$ saline at full and 2/3 maintenance; $0.18 \%$ saline at full and $2 / 3$ maintenance); the fall in sodium was smaller in the $2 / 3$ maintenance groups although not statistically significant $(p=0.12)$. Of note, the net positive fluid balance (i.e., total fluid intake minus total measured fluid output) in the $0.18 \%$ saline at 2/3 maintenance group was lower than all other groups, though also not statistically significant. Finally, Neville et al. [7] randomized 124 postoperative patients into 4 groups with saline contents of $0.9 \%$ at full and half maintenance rate and $0.45 \%$ at full and half maintenance rate. They noted a greater drop in serum sodium among those in the $0.45 \%$ saline groups as compared to the $0.9 \%$ saline groups $8 \mathrm{~h}$ after randomization and 
therefore concluded that iatrogenic hyponatremia was more attributable to the saline content than to the rate of administration. However, the authors note that this discrepancy nearly disappeared by $24 \mathrm{~h}$ because many in the $0.9 \%$ saline group at full maintenance rate developed hyponatremia between 8 and $24 \mathrm{~h}$. They proposed that excess fluid combined with desalination in a setting of persistent non-osmotic stimulation of increased ADH secretion may contribute to this phenomenon. They suggested that decreasing the rate of maintenance fluids and not increasing the saline content may be wise when prolonged IV fluids are required.

The rate of maintenance fluid administration is particularly important because of our current understanding of the physiologic reasons as to why hypotonic fluids contribute to iatrogenic hyponatremia. Specifically, many, if not most, hospitalized children and essentially all postoperative patients have conditions (Table 1) that lead to non-osmotic stimuli for ADH release that will lead to preferential retention of free water, which will thereby decrease serum sodium concentrations. Thus, administration of hypotonic fluids may be exceeding a patient's capacity to manage increased volumes of free water in the presence of such conditions. Children with pituitary dysfunction leading to increased levels of ADH secretion despite lack of osmotic or non-osmotic stimuli carry the diagnosis of syndrome of inappropriate antidiuresis (SIAD), previously referred to as syndrome of inappropriate antidiuretic hormone (SIADH). In such children, the primary approach to prevent and treat hyponatremia (in addition to treating the underlying cause) is fluid restriction [16], generally to 2/3 maintenance rates and further titration based on changes in serum sodium. Treatment of SIAD with isotonic fluid (e.g., $0.9 \%$ saline) is not typically recommended as increases in the serum sodium are usually modest, and some patients actually have decreased serum sodium if urine sodium is more concentrated than $0.9 \%$ saline $[17,18]$. Additionally, in the setting of non-osmotic stimuli for ADH release, isotonic fluids may mask SIAD and lead to fluid overload; Khan et al. [9] followed the serum and urine electrolytes of 40 children who underwent spinal fusion surgery, a known nonosmotic stimulus for ADH secretion, and were placed on isotonic maintenance fluids postoperatively. During their postoperative course, $32 \%$ developed signs of SIAD in the absence of hyponatremia including decreased serum osmolality, increased urine osmolality, high fractional excretion of sodium, low urine output, high urine sodium, and increased ADH levels. Those with SIAD had significantly greater fluid intake $(p=0.013)$ and net positive fluid balance ( $p=$ 0.008 ) along with increased postoperative weight gain ( $3.9 \mathrm{vs.} 2.5 \mathrm{~kg}, p=.058$ ), as well as dilutional anemia. Together, these findings suggest that ubiquitous use of isotonic maintenance fluids may lead to fluid overload and associated consequences [19-22].

\section{Algorithm for Initial Maintenance Fluid Choice}

In the setting of rapidly evolving research on the subject, we propose an algorithm for initial maintenance fluid choice for pediatric patients being admitted to the general pediatric ward based on three key features of the individual patient's physiology: intravascular volume status at the time of initiating maintenance intravenous fluid (IVF), serum sodium in those who 
Table 1. Common pediatric conditions that increase the risk of increased ADH secretion

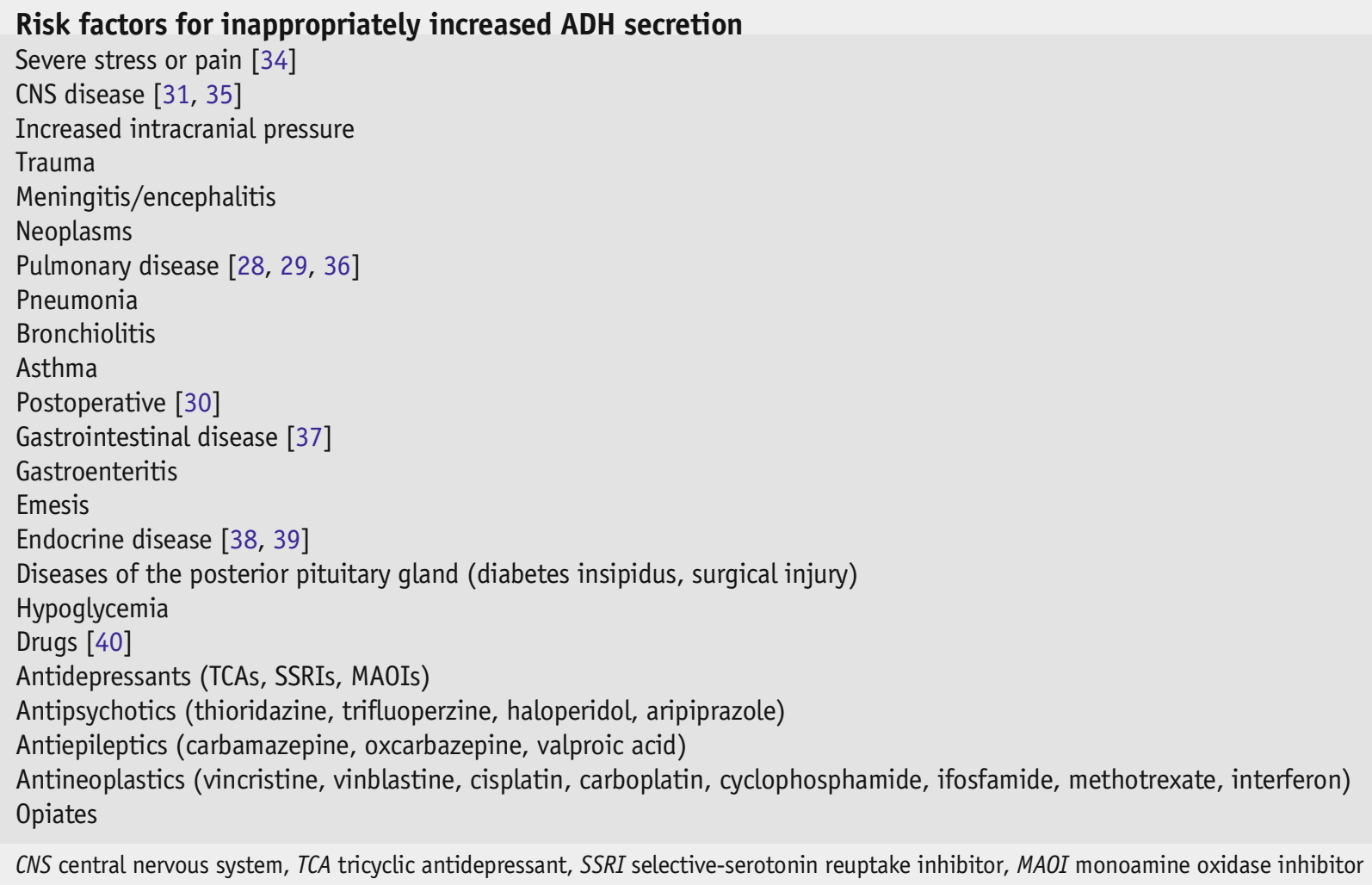

required volume resuscitation, and the likelihood of increased ADH levels from co-existent conditions (Fig. 1). We use an approach that clearly and separately identifies a patient's need for volume expansion (e.g., dehydration) from maintenance fluid needs and from ongoing losses. In this approach, completely or nearly completely re-establishing euvolemia prior to initiating maintenance IVF therapy is recommended.

\section{Assessment of Intravascular Volume and Initial Rehydration Therapy}

A complete discussion of techniques for assessment of intravascular volume in children is beyond the scope of this review. However, initial assessment of volume status is an important indicator of the underlying physiology of the patient when making maintenance fluid choices. Additionally, frequent reassessment of volume status over the course of maintenance therapy and appropriate repletion in addition to maintenance fluid is paramount to a safe IV fluid strategy.

Ideal assessment of intravascular volume and fluid-responsiveness is achieved through dynamic measurements such as pulse pressure variation, inferior vena cava diameter variation, echocardiography, or other techniques

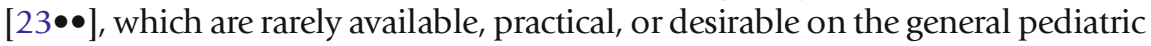
ward. Among patients with a known baseline healthy weight, the difference in total body water can be estimated, noting that each $1 \mathrm{~kg}$ difference corresponds to $1 \mathrm{~L}$ of fluid. In the absence of known pre-illness weight, multiple clinical 


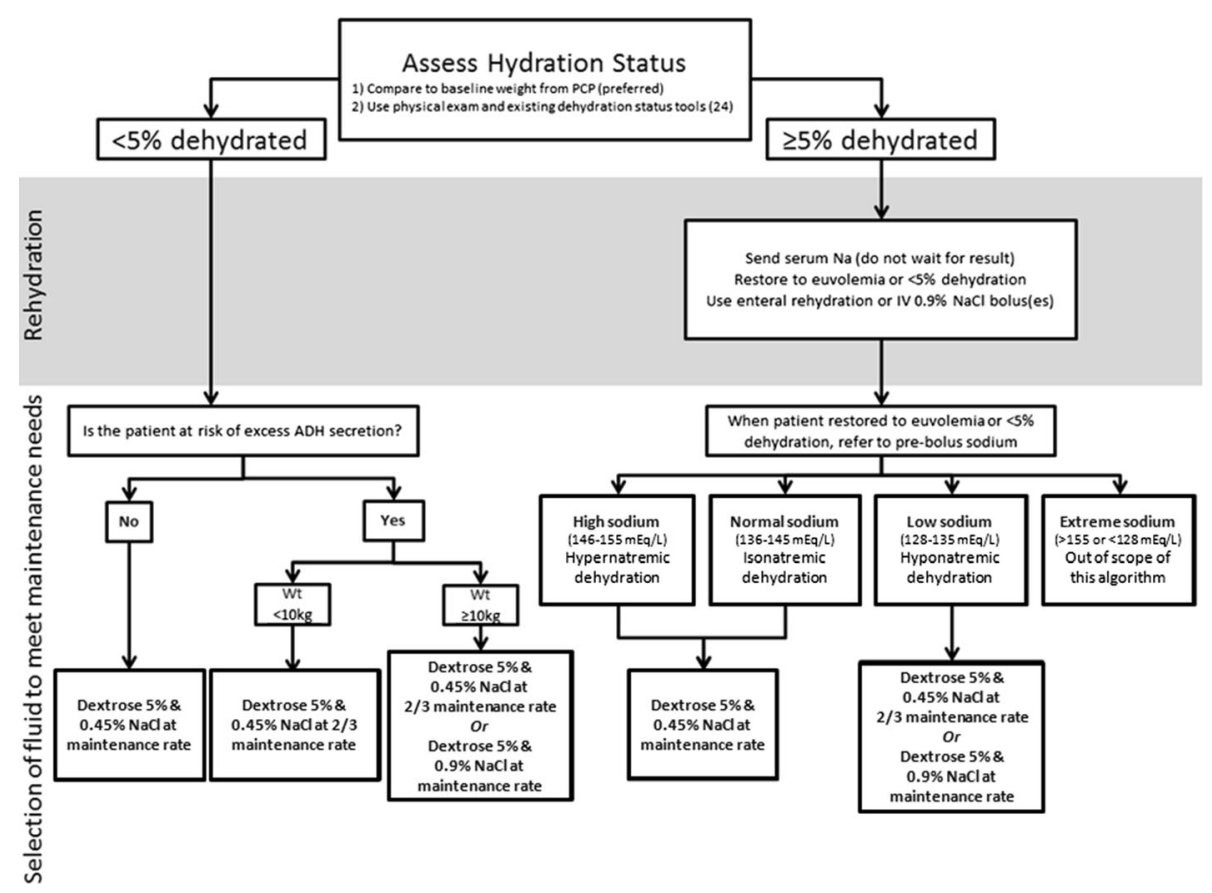

Fig. 1. Algorithm for initial selection of the rate and composition of maintenance IV fluids in children admitted to the general pediatric ward.

scores [24] based on physical examination findings, have been validated to yield an estimation of percent dehydration, which, when multiplied by the patient's weight, can be used to quantify the volume of missing fluid.

Enteral rehydration is preferred in mild-moderately dehydrated children when possible $[25,26]$. In those who require IV fluid resuscitation, the ideal type of fluid is controversial and beyond the scope of this review, but an isotonic fluid is typically deemed optimal for intravascular volume replacement, and $0.9 \%$ saline is the most widely used based on its low cost and overall efficacy [26]. Dextrose-containing isotonic solutions in patients with gastroenteritis have been shown to hasten ketone clearance [27].

\section{Maintenance Fluids in Euvolemic Patients}

Many hospitalized children have conditions associated with increased ADH secretion. Among these are surgery, central nervous system disease, pulmonary disease, acute pain, vomiting, and certain medications, which enhance either the release or effect of ADH [13, 28-32] (Table 1). It is believed that it is these patients who are at greatest risk for hyponatremia as a result of increased retention of free water, and their maintenance fluid choice should be selected accordingly. In these patients, dextrose 5 and $0.45 \%$ saline infused at $2 / 3$ the typical maintenance rate limits the amount of free water. In patients with increased $\mathrm{ADH}$, this helps avoid hyponatremia, while simultaneously limiting the total solute load, which decreases the risk for hyperchloremic metabolic acidosis and fluid overload. It seems likely that many of the cases of iatrogenic hyponatremia observed in the studies listed above are a result of administration 
of hypotonic fluids at a rate or total volume that exceeded the patient's ability to excrete free water when there were increased ADH levels.

\section{Maintenance Fluids in Patients who Present With Intravascular Volume Depletion}

Intravascular volume depletion serves as a non-osmotic stimulus of appropriate secretion of $\mathrm{ADH}$, but different patients will have varying amounts of water and solute losses. Thus, stratifying patients by initial serum sodium at presentation is a useful strategy for choosing maintenance fluids after euvolemia is restored. In those patients who are restored to euvolemia and have a normal or high serum sodium, the amount of sodium in isotonic fluids likely exceeds what is necessary to replete the sodium losses, risking the development of hypernatremia. As a result, hypotonic IV fluids (D5 $0.45 \%$ saline) at standard maintenance rates would be most appropriate. If the initial serum sodium is above $155 \mathrm{mEq} / \mathrm{L}$, slow correction with even more hypotonic fluids (D5 $0.2 \%$ saline) might be indicated. In contrast, those with hyponatremia at presentation have a relative free water excess and are at higher risk for worsening hyponatremia from excess free water in maintenance fluids. After restoration to euvolemia using isotonic fluids, $5 \%$ dextrose with $0.45 \%$ saline run at $2 / 3$ maintenance or $5 \%$ dextrose with $0.9 \%$ saline run at full maintenance may both be appropriate choices, depending on the patient's risk for fluid overload. That is, there is a higher risk of developing fluid overload with $5 \%$ dextrose with $0.9 \%$ saline infused at a full maintenance rate.

For those presenting with intravascular volume depletion in whom checking electrolytes is not indicated (e.g., asthma exacerbation with increased insensible losses from tachypnea improving with appropriate therapy), treating as described above for euvolemic patients along with assessment for risk of SIAD as well as frequent reassessments of hydration status and accounting for ongoing losses would be a sensible strategy.

Ongoing Losses

Patients with ongoing losses (e.g., vomiting, diarrhea) should have the volume and type of losses quantified and replaced as needed. Replacing the lost fluid with IV fluids of similar tonicity is least likely to lead to electrolyte disturbances [33]. These fluids should not be counted as part of the maintenance fluids but rather should be given separately and in frequent intervals in an attempt to maintain euvolemia.

\section{Conclusion}

Hyponatremia in the pediatric patient is a rare but potentially serious condition that can lead to seizures, encephalopathy, and even death. Literature over the previous decade has highlighted the development of hyponatremia in hospitalized children likely exacerbated by administration of hypotonic fluids beyond the patient's capacity to excrete free water. However, recommending the universal transition to isotonic maintenance fluids may oversimplify the pathophysiology unique to each patient and lead to different harms, in particular fluid overload from excess solute. Instead, it is more appropriate to ensure that intravascular volume depletion is properly appreciated and addressed and that those patients in whom increased ADH secretion is a risk are properly identified. A standardized 
approach to stratifying and categorizing patients in order to select appropriate maintenance fluids will serve as a cornerstone for strengthening the evidence base in this important field. We therefore recommend that future research on the impact of maintenance fluids focus on stratifying patients by likelihood of increased ADH levels, assessing balance measures associated with excessive isotonic fluid intake (particularly fluid overload and hyperchloremic metabolic acidosis), and gathering more data in understudied populations such as medical patients admitted to the general pediatric ward.

\section{Compliance With Ethics Guidelines}

\section{Conflict of Interest}

Jonathan B. Edelson declares that he has no conflict of interest. Evan W. Orenstein declares that he has no conflict of interest. Lisa B. Zaoutis declares that she has no conflict of interest. Lawrence Copelovitch declares that he has no conflict of interest.

\section{Human and Animal Rights and Informed Consent}

This article does not contain any studies with human or animal subjects performed by any of the authors.

\section{References and Recommended Reading}

Papers of particular interest, published recently, have been highlighted as:

- Of importance,

- Of major importance

1. Jain S, Elon LKM, Johnson BA, Frank G, DeGuzman M. Physician practice variation in the pediatric emergency department and its impact on resource use and quality of care. Pediatr Emerg Care. 2010;26(12):902-8.

2. Freeman M, Ayus J, Moritz M. Maintenance intravenous fluid prescribing practices among paediatric residents: Maintenance intravenous fluid prescribing practices. Acta Paediatr. 2012;101(10):e465-8.

3. Holliday MA, Segar WE. The maintenance need for water in parenteral fluid therapy. Pediatrics. 1957;19(5):823-32.

4. Wang J, Xu E, Xiao Y. Isotonic versus hypotonic maintenance IV fluids in hospitalized children: a metaanalysis. Pediatrics. 2014;133(1):105-13.

This meta-analysis, which examined 10 randomized control trials of pediatric patients receiving intravenous fluids, concluded that isotonic maintenance fluids were safer for pediatric patients than hypotonic maintenance fluids due to the risk of hyponatremia associated with hypotonic fluids.

5.•• Foster BA, Tom D, Hill V. Hypotonic versus isotonic fluids in hospitalized children: a systematic review and meta-analysis. J Pediatr. 2014;165(1):163-9.

This systematic review and meta-analysis examined 10 randomized control trials of hospitalized children receiving IV fluids and demonstrated an increased relative risk of hyponatremia in those patients receiving hypotonic fluids in comparison with those who received isotonic fluids. However, the authors noted in their thoughtful discussion that randomized studies were mostly performed in ICU settings or with post-operative patients and that few comparisons were done between isotonic fluid and $0.45 \%$ saline.

6. Kannan L, Lodha R, Vivekanandhan S, Bagga A, Kabra SK, Kabra M. Intravenous fluid regimen and hyponatraemia among children: a randomized controlled trial. Pediatr Nephrol. 2010;25(11):2303-9.

7. Neville KA, Sandeman DJ, Rubinstein A, Henry GM, McGlynn M, Walker JL. Prevention of hyponatremia during maintenance intravenous fluid administration: a prospective randomized study of fluid type versus fluid rate. J Pediatr. 2010;156(2):313-9.

8. Yung M, Keeley S. Randomised controlled trial of intravenous maintenance fluids. J Paediatr Child Health. 2009;45(1-2):9-14.

9. Khan I, Zimmerman B, Brophy P, Kamath S. Masking of syndrome of inappropriate antidiuretic hormone 
secretion: the isonatremic syndrome. J Pediatr. 2014;165(4):722-6.

10. Williams EL, Hildebrand KL, McCormick SA, Bedel MJ. The effect of intravenous lactated Ringer's solution versus $0.9 \%$ sodium chloride solution on serum osmolality in human volunteers. Anesth Analg. 1999;88(5):999-1003.

11. Skellett S, Mayer A, Durward A, Tibby SM, Murdoch IA. Chasing the base deficit: hyperchloraemic acidosis following $0.9 \%$ saline fluid resuscitation. Arch Dis Child. 2000;83(6):514-6.

12. Brandstrup B, Tønnesen H, Beier-Holgersen R, Hjorts $\varnothing$ $\mathrm{E}$, Ørding $\mathrm{H}$, Lindorff-Larsen $\mathrm{K}$, et al. Effects of intravenous fluid restriction on postoperative complications: comparison of two perioperative fluid regimens: a randomized assessor-blinded multicenter trial. Ann Surg. 2003;238(5):641-8.

13. Moritz ML, Ayus JC. Prevention of hospital-acquired hyponatremia: a case for using isotonic saline. Pediatrics. 2003;111(2):227-30.

14. Duke T, Molyneux EM. Intravenous fluids for seriously ill children: time to reconsider. Lancet. 2003;362(9392):1320-3.

15. Wales HI, Direct NHS. Alert. 2007 [cited 2014 Sep 30]; Available from: http://pmmp.cnki.net/Resources/ CDDPdf/evd\%5Cbase\%5CNeLH\%20Guidelines\% 20Finder\%5C\%E4\%B8\%B4\%E5\%BA\%8A\%E5\%AE\% 9Е\%Е8\%B7\%B5\%E6\%8C\%87\%E5\%8D\%97\% 5C2639.pdf

16. Spasovski G, Vanholder R, Allolio B, Annane D, Ball S, Bichet D, et al. Clinical practice guideline on diagnosis and treatment of hyponatraemia. Eur J Endocrinol. 2014;170(3):G1-47.

17. Musch W, Decaux G. Treating the syndrome of inappropriate $\mathrm{ADH}$ secretion with isotonic saline. QJM Mon J Assoc Physicians. 1998;91(11):749-53.

18. Steele A, Gowrishankar M, Abrahamson S, Mazer D, Feldman RD, Halperin ML. Postoperative hyponatremia despite near-isotonic saline infusion: a phenomenon of desalination. Ann Intern Med. 1997;126(1):20-5.

19. Foland JA, Fortenberry JD, Warshaw BL, Pettignano R, Merritt RK, Heard ML, et al. Fluid overload before continuous hemofiltration and survival in critically ill children: a retrospective analysis. Crit Care Med. 2004;32(8):1771-6.

20. Sutherland SM, Zappitelli M, Alexander SR, Chua AN, Brophy PD, Bunchman TE, et al. Fluid overload and mortality in children receiving continuous renal replacement therapy: the prospective pediatric continuous renal replacement therapy registry. Am J Kidney Dis. 2010;55(2):316-25.

21. Arikan AA, Zappitelli M, Goldstein SL, Naipaul A, Jefferson LS, Loftis LL. Fluid overload is associated with impaired oxygenation and morbidity in critically ill children. Pediatr Crit Care Med. 2012;13(3):253-8.

22. Goldstein SL. Fluid management in acute kidney injury. J Intensive Care Med. 2012;29(4):183-9.
23.• Davison D, Basu RK, Goldstein SL, Chawla LS. Fluid management in adults and children: core curriculum 2014. Am J Kidney Dis.

2014;63(4):700-12.

This paper reviews the fundamental physiologic principles of volume distribution, discusses advantages and disadvantages of crystalloid and colloid, evaluates targets of fluid resuscitation and assessment of those targets, explains measurement devices used to assess intravascular volume status, and finally assesses the adverse effects of excessive fluid administration across organ systems. While many of the measurement devices that appear in this article are not practically used on the general pediatric ward, the concepts reviewed remain a cornerstone to appropriate fluid management.

24. Jauregui J, Nelson D, Choo E, Stearns B, Levine AC, Liebmann $\mathrm{O}$, et al. External validation and comparison of three pediatric clinical dehydration scales. PLoS One. 2014;9(5):e95739. Carlo WA, editor.

25. Spandorfer PR, Mace SE, Okada PJ, Simon HK, Allen $\mathrm{CH}$, Spiro DM, et al. A randomized clinical trial of recombinant human Hyaluronidasefacilitated subcutaneous versus intravenous rehydration in mild to moderately dehydrated children in the emergency department. Clin Ther. 2012;34(11):2232-45.

26. Niescierenko M, Bachur R. Advances in pediatric dehydration therapy. Curr Opin Pediatr. 2013;25(3):304-9.

27. Levy JA, Bachur RG, Monuteaux MC, Waltzman M. Intravenous dextrose for children with gastroenteritis and dehydration: a double-blind randomized controlled trial. Ann Emerg Med. 2013;61(3):281-8.

28. Dhawan A, Narang A, Singhi S. Hyponatraemia and the inappropriate ADH syndrome in pneumonia. Ann Trop Paediatr. 1992;12(4):455-62.

29. Poddar U, Singhi S, Ganguli NK, Sialy R. Water electrolyte homeostasis in acute bronchiolitis. Indian Pediatr. 1995;32:59-65.

30. Burrows FA, Shutack JG, Crone RK. Inappropriate secretion of antidiuretic hormone in a postsurgical pediatric population. Crit Care Med. 1983;11(7):527-31.

31. Cotton MF, Donald PR, Schoeman JF, Van Zyl LE, Aalbers C, Lombard CJ. Raised intracranial pressure, the syndrome of inappropriate antidiuretic hormone secretion, and arginine vasopressin in tuberculous meningitis. Childs Nerv Syst ChNS Off J Int Soc Pediatr Neurosurg. 1993;9(1):10-5.

32. Robertson G, Berl T. Pathophysiology of water metabolism. The Kidney. Philadelphia, PA: WB Saunders Co; 1996. p. 873-928

33. Hines EQ. Fluids and Electrolytes. The Harriet Lane Handbook. 19th ed. Philadelphia, PA: Elsevier Mosby; p. 271-92

34. Habib KE, Gold PW, Chrousos GP. Neuroendocrinology of stress. Endocrinol Metab Clin North Am. 2001;30(3):695-728. 
35. Jelski W, Laniewska-Dunaj M, Orywal K, Kochanowicz J, Rutkowski R, Szmitkowski M. The activity of alcohol dehydrogenase (ADH) isoenzymes and aldehyde dehydrogenase (ALDH) in the sera of patients with brain cancer. Neurochem Res. 2014 Oct 10

36. Iikura Y, Odajima Y, Akazawa A, Nagakura T, Kishida $\mathrm{M}$, Akimoto K. Antidiuretic hormone in acute asthma in children: effects of medication on serum levels and clinical course. Allergy Proc Off J Reg State Allergy Soc 1989;10(3):197-201.
37. Neville KA. High antidiuretic hormone levels and hyponatremia in children with gastroenteritis. Pediatrics. 2005;116(6):1401-7.

38. Robertson GL. Antidiuretic hormone. Normal and disordered function. Endocrinol Metab Clin North Am. 2001;30(3):671-94.

39. Baylis PH, Robertson GL. Rat vasopressin response to insulin-induced hypoglycemia. Endocrinology.

1980;107(6):1975-9.

40. Liamis $\mathrm{G}$, Milionis $\mathrm{H}$, Elisaf $\mathrm{M}$. A review of drug-induced hyponatremia. Am J Kidney Dis. 2008;52(1):144-53. 